\title{
Robust Object Tracking with Mutualism between Particle Filter and Geometric Active Contour
}

\author{
Lei Sun, Sun-an Wang, Jin-hua Zhang ${ }^{*}$ and Xiao-hu Li \\ School of Mechanical Engineering, Xi'an Jiaotong University, Xi'an 710049, \\ Shaanxi, China \\ sunly029@gmail.com,sawang@mail.xjtu.edu.cn, \\ jjshua@mail.xjtu.edu.cn,catlpx@sohu.com
}

\begin{abstract}
Robust object tracking is important in many real applications. This study proposes a novel robust object tracking algorithm that combines particle filter and geometric active contour. In contrast to conventional combinations, the proposed algorithm establishes mutualism, wherein the particle filter provides curve initialization for the active contour by using the alpha shape algorithm, and the active contour provides evolved boundary for particle filter classification. The refined particles are used to estimate the states which reduce the distance error. Discriminable object feature is proposed to enhance the tracking robustness, and prior information-based curve evolution is used to improve the accuracy of boundary segmentation. Comparable experiments are performed using a stand particle filter and continuously adaptive mean-shift methods. The proposed algorithm is found to be highly robust for long video sequences with non-linear motion, scale changes and clutter background.
\end{abstract}

Keywords: particle filter; geometric active contour; alpha shape; mutualism; robust object tracking

\section{Introduction}

Visual object tracking plays an important role in the field of computer vision. It has a variety of significant applications such as human-computer interfaces, road traffic control, video surveillance systems [1-3]. But it still remains a challenging problem. Because the background and object can be extremely complex and variable, especially in outdoor environments. Here, we mention some problems of object tracking such as illumination changes, presence of background clutter, scale changes, rotation, etc. In order to deal with them, many research efforts and attentions have been devoted. The solutions could be roughly classified into two categories: deterministic tracking and stochastic tracking.

Stochastic tracking approaches are based on the Bayesian estimation theory and often reduced to an estimation problem. Particle filter is the most representative method. It has gained interest for stochastic visual tracking for its intrinsic capability such as adapting to scale changes, tracking multiple hypotheses and coping with the nonlinearity and non-gaussian dynamic system [4].Although particle filter has many advantages, it can not handle local deformations of the deforming object and its topology change. And particle degeneration is another needed to solve. Geometric active contour belonging deterministic filed which can solve the above problems easily, are being research hot spot. Deterministic approaches usually reduce to an optimization problem. The definition of the cost function is a key issue. The common choice are Mean-shift, Continuously Adaptive

* Correspondiong Author 
Mean-shift(CamShift) and geometric active contour. One of the main drawbacks of standard MeanShift and CamShift tracking is that it is prone to tracking failures caused by objects with similar colors [5]. Recently level set-based active contour is widely used to extract object, which is first presented in literature [6]. Its basic idea is to evolve a curve, subject to constraints from a given image $u_{0}$, in order to detect objects in that image. Now the typical variant is $\mathrm{C}-\mathrm{V}$ region segmentation algorithm based on a reduced Mumford-Shah model and variational level set [7].

In this paper, object tracking is tackled in complex environments, including heavy clutters, low resolution image sequences and non-stationary camera, where the existence of multiple features or well object detection is not guaranteed. Particle filter is chose to estimate the object state vector which is non-gaussian and non-linearity. Geometric active contour method is implemented to handle local deformations of the deforming object and its topology change. And also to eliminate useless particles for resampling, maintaining particles diversity. Some other research works about combination have been carried out. In order to mark rapid motion cell boundary, Shen et. al., implement the particle filter to track biological cells and then evolve the cell boundary with contour initialization using previous state estimation [8]. Estimate the state of a system given a set of observations. The state refers to the positions and shape parameters of contours in this application. Rathi et. al., formulate a particle filtering algorithm in the geometric active contour framework that can be used for tracking moving and deforming objects [9]. The scheme combines the advantages of particle filtering and geometric active contours for dynamic tracking. They use the 6-dimensional affine parameters and the infinite dimensional contour of curves as the state, i.e., contour is a part of the state space. The previous contour is affined as the initial guess of the contour. Sun et. al., propose a refined particle method to track object contour, combining with a binary level set model [10]. It regards the particle represented by a rectangle in prediction step or active contour in update step as the state space. Although it avoids the re-initialized process of the level set function in each iteration as well as the cumbersome numerical realization, it uses the similar filter framework with literature [9].It still use the previous contour as the curve initialization guess contour. The evolution curve contour is implemented to compute the likelihood. Sandhua et. al., introduce a new similarity metric based on prediction theory to the particle filtering framework [11]. The state space consists of object's center and contour. Contour is evoluted with geometric active contour which incorporate a separate energy term to penalize large centric displacements. The initialization is determined by previous contour and the importance weight is updated by energy function. No matter the finite dimensional or infinite dimensional state space, the dimension of state space is high. High dimension state needs more particles for accurate filtering, which will compute expensively [12].And the curve is evolved to compute the likelihood with exponential function. Another approach is used to classify the particles with evolved contour. Luo et. al., use the particle filter to track object of interest in complex environment and then add the shape prior to the geometric active contour to evolve the curve in the particle weight-based sparse space [13]. Make one-class classification of particles with evolved contour. Although combination is implemented to improve the track performance, particles useful information is not deeply exploited. Also the contour evolution is depended on sparse space, it make no use of image information. It cannot obtain the real object contour despite of computing simply.

Inspired by the idea of Luo et. al., the current study proposes a new robust method that combines particle filter and active contour under non-ideal conditions. The proposed method thoroughly develops the position information of particles to generate initial guess contour for curve evolution and evolves the curve in an original image space instead of in a sparse space. We regard the combination as 'MutFilter'. Other techniques, such as the discriminable feature and prior information-based $\mathrm{C}-\mathrm{V}$, are used to track the objects robustly. The rest of this paper is organized as follows. Section 2 describes the preliminary 
theories, such as particle filter, geometric active contour and alpha shape. Section 3 describes the proposed object tracking method in detail. Section 4 presents the experimental analysis and the results of different algorithms. Section 5 draws the conclusion.

\section{Preliminary}

\subsection{Particle Filter}

In the sequential Bayesian estimation framework, the goal is to estimate the posterior probability density function (PDF) $p\left(x_{t} \mid Y_{t}\right)$, where $x_{t}$ is the state vector of a target at time t, and $Y_{t}=\left\{y_{1}, \ldots y_{t}\right\}$ is the collection of measurements (i.e., image frames) from time 1 to the current time t. This estimation is performed in two steps, namely, prediction and update. The prediction of future time $t$ can be expressed as a multidimensional integral.

Prediction:

$p\left(x_{t} \mid Y_{t-1}\right)=\int p\left(x_{t} \mid x_{t-1}\right) p\left(x_{t-1} \mid Y_{t-1}\right) d x_{t-1}$

Where $p\left(x_{t} \mid x_{t-1}\right)$ is the transitional PDF that is typically adopted in the target dynamic (motion) model. Using the new image $y_{t}$, the update step can be described as follows:

Update:

$p\left(x_{t} \mid Y_{t}\right)=\frac{p\left(y_{t} \mid x_{t}\right) p\left(x_{t} \mid Y_{t-1}\right)}{p\left(y_{t} \mid Y_{t-1}\right)}$

Where $p\left(y_{t} \mid x_{t}\right)$ is the measurement likelihood function that is adopted in the observation model. In general, the sequential Bayesian estimator has no closed-form analytic solution. Thus, the Monte Carlo method is implemented to generate approximations. In this framework, the posterior PDF at time $t$ is represented by a set of weighted particles $\left\{\omega_{t}^{n}, x_{t}^{n}\right\}_{n=1}^{N}$; that is,

$p\left(x_{t} \mid Y_{t}\right) \approx \sum_{n=1}^{N} \omega_{t}^{n} \delta\left(x-x_{t}^{n}\right)$

Where $q\left(x_{t} \mid x_{t-1}^{n}, y_{t}\right), w_{t}^{n}=\frac{w_{t}^{n}}{\sum_{j=1}^{N} \omega_{t}^{j}}$, and $w_{t}^{n}$ is given by

$w_{t}^{n}=w_{t-1}^{n} \frac{p\left(y_{t} \mid x_{t}^{n}\right) p\left(x_{t}^{n} \mid x_{t-1}^{n}\right)}{q\left(x_{t}^{n} \mid x_{t-1}^{n}, y_{t}\right)}$

Typically, the chosen proposal density is equal to the transitional density, that is, $q\left(x_{t}^{n} \mid x_{t-1}^{n}, y_{t}\right)=p\left(x_{t}^{n} \mid x_{t-1}^{n}\right)$. Equation (4) is thus simplified to $\omega_{t}^{n}=\omega_{t-1}^{n} p\left(y_{t} \mid x_{t}^{n}\right)$ which is called the bootstrap particle filter. The particle filter is initialized by generating a set of weighted particles from the prior density $p\left(x_{0}\right)$. Equation (4) provides a recursion for computing the set of weighted particles representing $p\left(x_{t} \mid Y_{t}\right)$ from those representing $p\left(x_{t-1} \mid Y_{t-1}\right)$ using a new measurement $y_{t}$. The samples are resampled according to their importance weights to generate a set of unweighted particles and to consequently avoid impoverishment. 


\subsection{Geometric Active Contour}

Despite the advantages provided by the particle filter, it still has some limitations, such as particle degeneracy and object deformation. Geometric active contour is one of the best methods that can be used to address such drawbacks. Geometric active contour can solve not only the degeneracy with resampling but also the deformation problem with curve evolution. In this study, we use the $\mathrm{C}-\mathrm{V}$ algorithm proposed in [7]. This algorithm can describe the curve evolution energy $E\left(C_{1}, C_{2}, \phi\right)$ as follows:

$$
\begin{aligned}
& E\left(C_{1}, C_{2}, \phi\right)=\mu \int_{\Omega} \delta(\phi(x, y))|\nabla \phi(x, y)| d x d y+\lambda_{1} \int_{\Omega}\left|u_{0}(x, y)-C_{1}\right|^{2} H(\phi(x, y)) d x d y \\
& +\lambda_{2} \int_{\Omega}\left|u_{0}(x, y)-C_{2}\right|^{2}(1-H(\phi(x, y))) d x d y
\end{aligned}
$$

Where $C_{1}, C_{2}$ and the Heaviside function $H(\phi)$ are given by:

$$
\begin{array}{cc}
C_{2}(\phi)=\frac{\int_{\Omega} u_{0}(x, y)(1-H(\phi(x, y))) d x d y}{\int_{\Omega}(1-H(\phi(x, y))) d x d y} & C_{1}(\phi)=\frac{\int_{\Omega} u_{0}(x, y) H(\phi(x, y)) d x d y}{\int_{\Omega} H(\phi(x, y)) d x d y} \\
\text { and } \quad H(\phi)= \begin{cases}1 & \phi \geq 0 \\
0 & \text { else }\end{cases} & \delta_{0}(\phi)=\frac{d}{d \phi} H(\phi)
\end{array}
$$

Where $u_{0}(x, y)$ is the image, and $\phi(x, y)$ is the level set function. They deduce the Euler-Lagrange equations for $\phi$ and minimize the energy $E\left(C_{1}, C_{2}, \phi\right)$ by performing the gradient descent using the following PDE:

$\frac{\partial \phi}{\partial t}=\delta_{\varepsilon}(\phi)\left[\mu \operatorname{div}\left(\frac{\nabla \phi}{|\nabla \phi|}\right)-\nu-\lambda_{1}\left(u_{0}-c_{1}\right)+\lambda_{2}\left(u_{0}-c_{2}\right)\right]=0$

in $(0, \infty) \times \Omega, \phi(0, x, y)=\phi_{0}(x, y)$ in $\Omega, \frac{\delta_{\varepsilon}(\phi)}{|\nabla \phi|} \frac{\partial \phi}{\partial \vec{n}}=0$ on $\partial \Omega$, where $\vec{n}$ denotes the exterior normal to the boundary $\partial \Omega$, and $\frac{\partial \phi}{\partial \vec{n}}$ denotes the normal derivative of $\phi$ at the boundary. The performance of the segmentation strongly depends on the extent to which the assumption of homogeneous image data is satisfied. To segment object accuracy, prior object model information is used in guiding the curve stopping at the correct boundary.

\subsection{Alpha Shape}

Although the geometric active contour can provide accurate object boundary for classifying the particles as either effective or outliers, it needs to be initialized with coarse boundary for evolution. Thus, the particles are used in initialization which we regard as mutualism mechanics. The particles are scattered around the object with likelihood weight and fixed position coordinate. First, we filter the weighted particles by the threshold and then determine the outer boundary of the convex hull consisting of particles that correspond to a fixed coordinate. The alpha shape method is used to construct the boundary [14]. This method uses a finite set of points (particles) and the $\alpha$ parameter in the plane to search for the boundary point and to connect this point to the uniquely built polygon.

Consider a set of points (particles positions in our case) $S=\left\{\left(x_{s}, y_{s}\right), s=1,2 \ldots N\right\}$. The alpha shape is a uniquely defined region that approximates its intuitive 'shape'. For the negative real, the alpha hull is the intersection of the closed complements of the discs of 
radius, each of which completely contains $S$ [15]. The alpha shape is the straight line graph formed by connecting the neighboring points on the boundary of the alpha hull; it essentially replaces the concave arcs of the alpha hull with lines. Figure 1 shows an example of the alpha shape. The alpha shape can be described qualitatively by rolling a hoop of radius around the edges of the point cloud [16]. If the hoop touches two points, and the hoop's interior does not contain additional points, then the line segment containing the two points is on the boundary of the alpha shape. The following describes the implementation of the alpha shape. The Delaunay triangulation is a set of triangles (simplexes) with vertices such that the triangle's circumcircle does not contain any point in $S$; the alpha shape is a subset of the Delaunay triangulation of $S$. The alpha complex is a set of all simplexes of the Delaunay triangulation that are in the interior of the alpha shape; its boundary is the alpha shape.

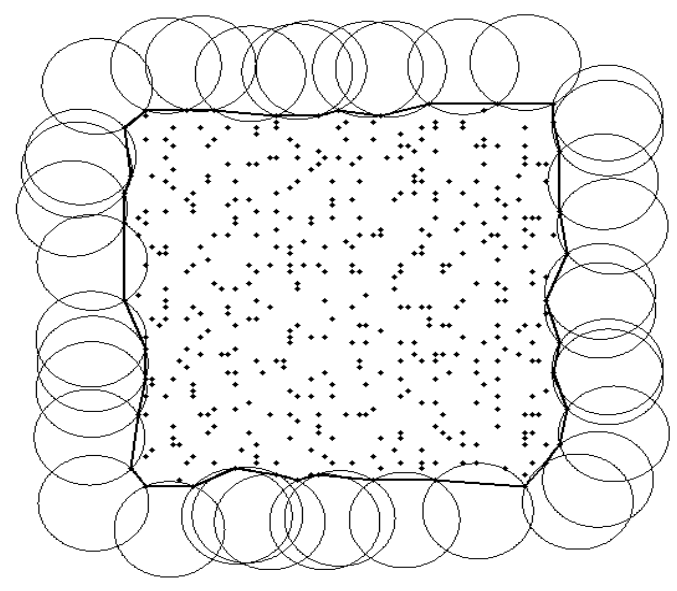

Figure 1. Alpha Shape Schematic

\section{Object Tracking}

\subsection{Object Histogram}

Color histogram is an important image feature that expresses overall image information and has good robustness against translation, rotation and deformation. This feature also involves simple computation. The histogram of an image can be described as follows:

$n_{b}=\frac{1}{M * N} \sum_{i=1}^{M} \sum_{j=1}^{N} \delta_{b}(i, j), \forall b=1,2 \ldots B$

Where $\delta_{b}(i, j)=1$ if the value at pixel location $(\mathrm{i}, \mathrm{j})$ falls into bin $\mathrm{b}$; otherwise, $\delta_{b}(i, j)=0$. From a probabilistic point of view, the normalization of a histogram results in a function that is most akin to the PDF of the data. A histogram can be used to describe the statistical properties of a given distribution. Thus, the most common features of a histogram are used to express the similarities in an object tracking task. Similarities between two different histograms, $n_{b}$ and $n_{b}^{\prime}$, can be computed using various techniques, such as histogram intersection or the Bhattacharyya coefficient. In this study, we adopt the Bhattacharyya coefficient $\rho_{b}^{h}\left(n_{b}, n_{b}^{\prime}\right)=\sum_{b=1}^{B}\left(n_{b} n_{b}^{\prime}\right)^{0.5}$ as the similarity measure. However, a histogram is sensitive to changes in contrast and lacks information about how a color is spatially distributed in an image, particularly when the scene contains other objects characterized by a color distribution similar to that of the object of interest. Thus, the consideration of a discriminate feature is inevitable for robust tracking. 


\subsection{Discriminate Color based on $\mathrm{C}-\mathrm{V}$}

Tracking success or failure depends primarily on how distinguishable an object is from its surroundings. If the object has low contrast or is camouflaged, then it may not be correctly segmented out and tracked. However, the sub-target of the object's interior becomes highly distinguishable from the background. Thus, the most discriminate local region should be determined to model the object robustly. Let Ro be the region that corresponds to the target with centre $\mathrm{C}$, width $\mathrm{w}$, and height $\mathrm{h}$. Let $\mathrm{Rb}$ be the region that corresponds to the background with centre $\mathrm{C}$, width $2 \mathrm{w}$, and height $2 \mathrm{~h}$, excluding the region Ro. The bins are set to six to describe the region color feature. The following six colors are often used, particularly in back projection showing a colored image: red, green, blue, cyan, magenta, and yellow. These color regions identify the homogeneous regions of the $\mathrm{C}-\mathrm{V}$ algorithm, thereby improving segmentation performance. To determine the most discriminate region, we first obtain six bins of the numerical quantity histogram from the back projection image instead of the probability histogram used in most image processing tasks. For all candidate feature spaces, the space with the maximum block ratio value is determined as the feature space and chosen as the color block region.

ind $x=\underset{i \in \text { bins }}{\operatorname{argmax}} \frac{\operatorname{obhist}(i)}{\operatorname{bghist}(i)} \quad$ bins $=[1,2, \ldots 6]$

Where obhist and bghist represent the target frequency histogram and background frequency histogram, respectively (Figure 2).

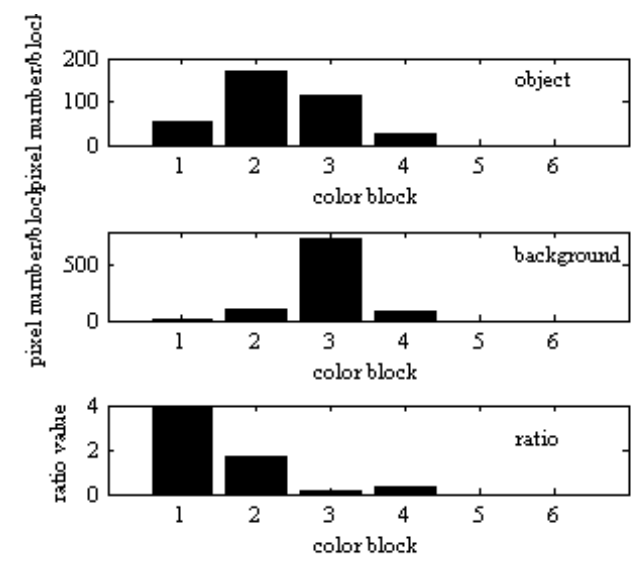

Figure 2. Discriminate Histogram Ratio

\subsection{Boundary initialization}

Initial contour must be given to evolve the curve to the object edge. An initial contour that closely resembles the real contour indicates the former's superior performance, which can be demonstrated by a low evolution time and a highly accurate contour. The weighted particles are used to build the coarse contour. A large particle weight indicates the close proximity of the particle to the object centre. Thus, heavily weighted particles may be found in the neighborhood of the real object edge. Fig 3 shows the rectangle region of the object and the scattered particles. Assume that the object color is uniformly distributed. Object histogram $n_{0}$ and candidate histogram $n_{c}$ can be obtained using Equation (7). When the particle is on the boundary (left dot on the rectangle), that is, 
$\rho_{b}^{h}\left(n_{o}, n_{c}\right)=\sum_{b=1}^{B} \sqrt{n_{o} n_{c}}=\sum_{b=1}^{B} \sqrt{0.5 n_{o} * n_{o}}=\frac{\sqrt{2}}{2}$

When the particle is on the corner (right dot on the rectangle), that is,

$n_{c}=0.25 * n_{o}, \rho_{b}^{h}\left(n_{o}, n_{c}\right)=0.5$

In real applications, the object may take any shape. Thus, the threshold described above is used as a reference only. In this study, we choose $\rho=0.5$ to filter the particles. The remaining particles are used to determine the coarse contour around the object using the alpha shape method. The alpha parameter is chosen as follows. According to the particle distribution variance, we set $\alpha=\sigma^{2} / 2$ to facilitate the adaptive approach of the polygon to the object boundary. Figure 4 shows the procedure of curve initialization by alpha shape and final evolved contour.
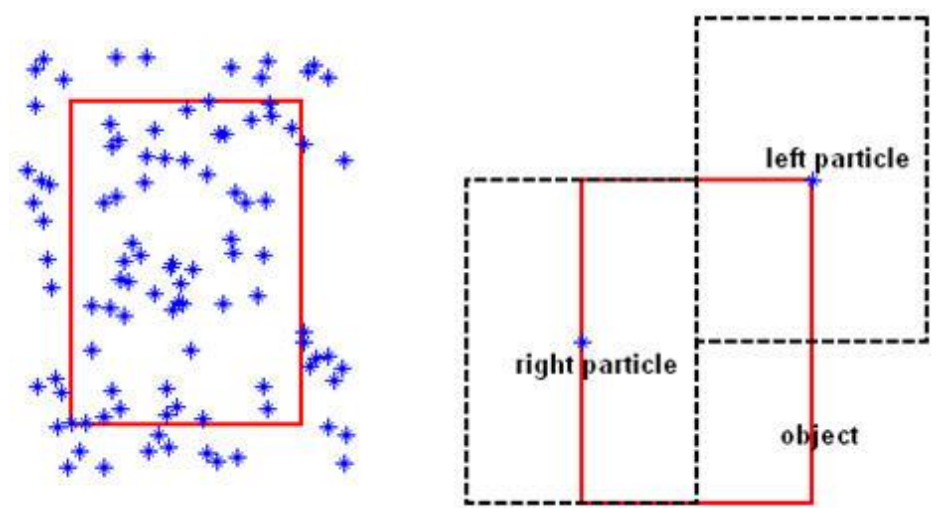

Figure 3. Particles Positions Distributed

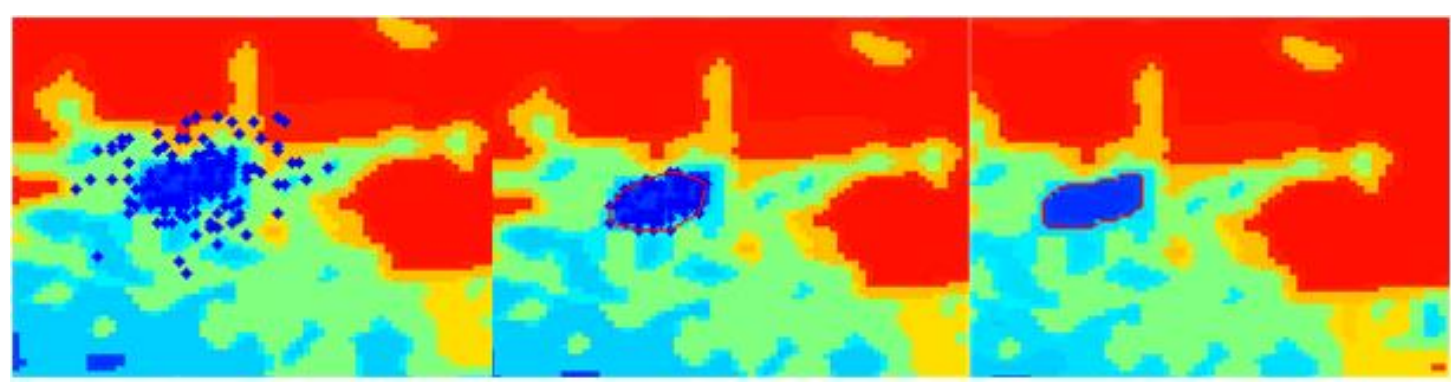

Figure 4. Evolution after Alpha Shape

\subsection{C-V Guide Information}

To segment accurately under a cluttered environment, prior knowledge is used in guiding the curve evolution. Shape prior is introduced to the level set representation for object extraction under the occlusion condition [17-18]. The implicit premise is that the contour can be segmented well. In a cluttered environment, the target edge blends with the background pixels. Thus, the contour is deformed severely and discriminated from the prior shape. Thus, even when the shape prior is used to guide the object segmentation, the result will be different from the target contour because of image force. According to Equation (5), the curve definition is the entire image, $C_{1}$ and $C_{2}$ will also be in the image. The curve will be global minimized by the energy function with the homogeneous region. However, the global region contains many interference factors, and the values of $C_{1}$ and $C_{2}$ change dynamically at each iteration. The segmentation performance relies on the accuracy of $C_{1}$ 
and $C_{2}$. Thus, the static average of the limitation region is used to compute the average value instead of $C_{1}$ and $C_{2}$. The determined values of $C_{1}$ and $C_{2}$ can improve the object contour. Figure 5 shows a schematic diagram of the limitation region, where $C_{1}$ is the average value of the local contour, and $C_{2}$ is the average value that is twice that of the contour excluding the local contour.

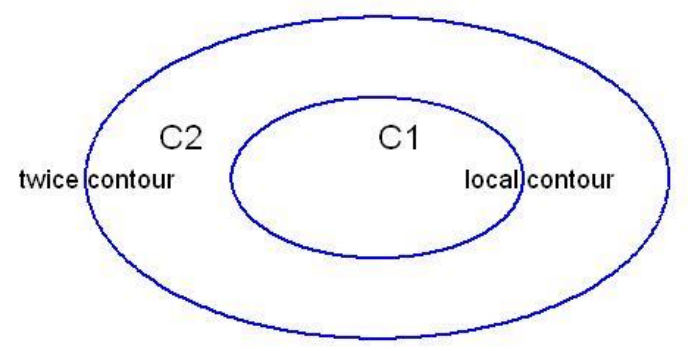

Figure 5. Schematic Diagram of Limitation Region

\subsection{Likelihood with Multiple Cues}

The classical $\mathrm{C}-\mathrm{V}$ model results in a significant amount of redundant contours. In this case, the target may undergo splitting and merging. Thus, the choice for the most similar target contours is based on the fusion of multiple cues. Many features, such as mean value, phi-based histogram and contour and velocity vector, are used to compute the mixture likelihood. First, we use the Hausdorff distance to measure the similarity between the current contour and the object contour.

$D(A, B)=\max (h(A, B), h(B, A))$

Where $h(A, B)=\max _{a \in A} \min _{b \in B}\|a-b\|$, and $h(B, A)=\max _{b \in B} \min _{a \in A}\|b-a\|$. See literature [19] for details.

The contour likelihood can be defined as follows:

Lcontour $\left(y_{\text {contour }} \mid x\right) \propto \exp \left(-\frac{D\left(C_{c a n}, C_{o b j}\right)}{2 \sigma_{C}^{2}}\right)$

Where $C_{c a n}$ and $C_{o b j}$ denote the candidate contour and the object contour, respectively. The mean value and phi-based histogram are computed using the candidate contour. Inspired by the principle of kernel, we use the signed distance function (SDF) instead of kernel distance to reduce the influence of the boundary pixels, as a contour can take any shape, and the calculation of kernel distance is inconvenient. After integrating the SDF into each pixel using Equation (13), we compute the mean value likelihood and histogram likelihood using Equations 14 and 15.

$$
\begin{aligned}
& u_{0}(x, y)=u_{0}(x, y) * \exp (\phi(x, y)) \\
& L_{\text {mean }}\left(y_{\text {mean }} \mid x\right) \propto \exp \left(-\frac{{\overline{C_{1}}}^{2}}{2 \sigma_{\text {mean }}^{2}}\right)
\end{aligned}
$$

where $\overline{C_{1}}=\operatorname{mean}\left(u_{1}(x, y)\right)$, in which $u_{1}(x, y)$ is the inside contour. 
$L_{\text {hist }}\left(y_{\text {hist }} \mid x\right) \propto \exp \left(-\frac{1-\rho}{2 \sigma_{\text {hist }}^{2}}\right)$

Where $\rho$ is the Bhattacharyya coefficient of the histogram.

When the target merges into the similarity background and cannot be discriminated, the target and the background are tracked as a whole. However, when the target goes out of the background, it undergoes splitting from the background. The velocity vector is used to detect splitting and to track the target. The mixture region expands as the target splits from the background. The orientation of target splitting can be determined using the expansion region of the target. Once the mixture region is split by more than two targets, splitting detection occurs in all current contours that intersect previous contours. The sub-target velocity vector is filtered via mean smoothing with previous vectors. This vector is used to compute the similarity with the target object.

$L_{\text {velocity }}\left(y_{v} \mid x\right) \propto \exp \left(\operatorname{sign}(\vec{v} \bullet \overrightarrow{m v}) * \exp \left(-\frac{\frac{\vec{v} \bullet \overrightarrow{m v}}{\|m \overrightarrow{m v}\|}}{2 \sigma_{v}^{2}}\right)\right.$

Where $\vec{v}$ is the current velocity vector, and $\overrightarrow{m b}$ is the average value of the previous $N$ velocity vector.

By combining the velocity vector likelihood with the three likelihoods above using Equation (17), the final likelihood can be obtained, and the target can be determined using the max likelihood.

$L\left(y_{t} \mid x_{t}\right)=L_{\text {contour }} * L_{\text {velocity }} * L_{\text {hist }} * L_{\text {mean }}$

\subsection{Contour-Based Particle Resampling}

Conventional resampling algorithms, such as systematic resampling or sampling importance resampling, impose negative influence when the feature is not discriminated. Resampling only emphasizes on particle weight and does not consider other useful information, such as the allocation, scattering, and shape of the object. Occasionally, the particle with low weight may be more important than that with high weight. Inspired by the work that constructed on-class classification, we attempt to model one class of objects and distinguish it from other possible outliers [20]. The contour is used to distinguish the target particles inside the contour from the outlier particles outside the contour (Figure 6). To improve the diversity of the discrete particles, the non-parameter-based Parzen density estimator is adopted in estimating the continue state distribution of target particles [21].

$p_{N}(x)=\frac{1}{N} \sum_{j=1}^{N} \frac{1}{h^{N}} K\left(\frac{x-x_{j}}{h^{N}}\right)$

Where $\mathrm{N}$ is the sample number, $\mathrm{K}$ is the kernel function and $h^{N}$ is the bandwidth.

In Equation (18), a general kernel approach instead of the Dirac delta kernel approach is used to approximate effectively the posterior distribution. Stratified resampling method is used to resample particles from the continuous state space as well as to prevent particle impoverishment. 


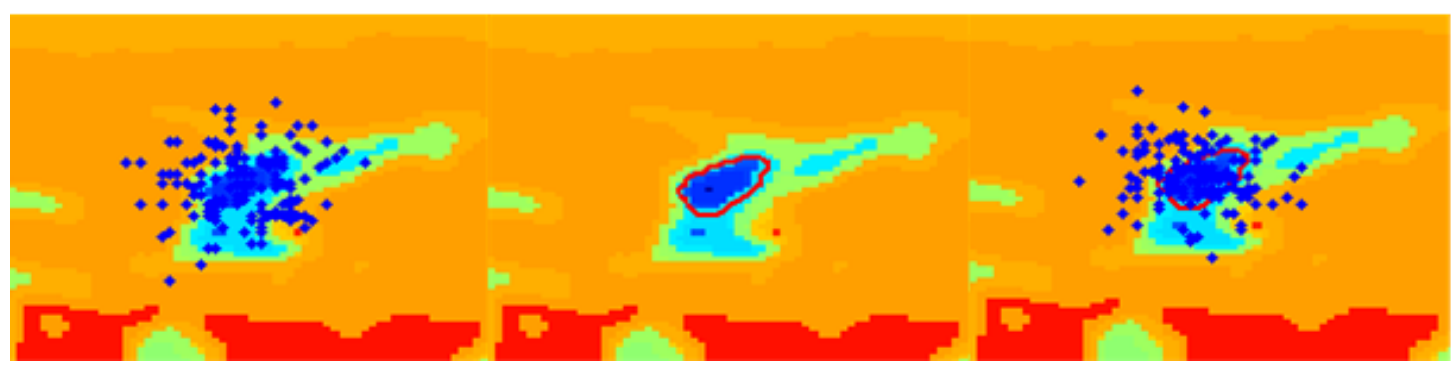

Figure 6. Classification by Contour

\section{Experiment and Discussion}

The proposed method is applied to track objects in a complex environment. Standard particle filters and CamShift algorithms in real digital video with 760 frames are used in the contrast experiments. The results highlight the superior performance of the proposed algorithm. In the experiment, all the values of the feature space are normalized into the closed interval [0 511]. The key parameters are as follows: number of particles $N_{p}=150$, histogram bins $B=512$, region coefficient $\lambda_{1}=\lambda_{2}=1$, length coefficient $\mu=1$ and iterate number iter $\_n=200$. We compare the algorithm location (AT) with the manually labeled 'ground truth'(GT) locations of the object. The following parameters for performance measurement are defined.

- $\operatorname{err}(t)$ represents the Euclidean distance and is equal to $\|G T(t)-A T(t)\|_{2}$, where $\mathrm{t}$ is time, and $G T(t)$ and $A T(t)$ are the location coordinate vectors that correspond to $t$.

- $\bar{Q}$ represents the average error distance and is equal to $\frac{1}{N} \sum_{t=1}^{N} \operatorname{err}(t)$, where $\mathrm{N}$ is the number of successfully tracked frames.

- $R_{\text {sucess }}$ denotes the total number of frames divided by the number of successfully tracked frames.

- $N_{\text {loss }}$ denotes the number of frames that cannot be tracked.

The low resolution of video data $(320 * 240)$, the small size of the helicopter being tracked and the dramatic motion of the camera make the tracking difficult. The video sequence is a complex environment for object tracking in two aspects. First, no appropriate features, such as color, edge and/or texture, can distinguish the helicopter from the trees in the background. Second, the helicopter keeps floating over certain positions for many times. All floating movements last for a non-trivial duration, thus making the dynamics of motion useless. For example, when the helicopter emerges from the cluttered leaves, it floats in front of a background clutter and then slowly moves away after 40 frames.

From the 200th frame to the 250th frame, the image is zoomed gradually, thus changing the scale. The particle filter, as well as the CamShift and the proposed algorithm, is naturally able to deal with scale changes. In the CamShift algorithm, the rectangle region of the initial object is small; otherwise, tracking diverges and enlarges the region. In this case, many background pixels influence the peak probability of BP. Upon entering the clutter leaves, the CamShift tracker gets stuck on the local optimum; the tracking region then loses the real object after much iteration (Figure 7). The peak of the probability distribution of BP does not involve color composition, thus resulting in the departure of the target object. The PF method is influenced by a cluttered environment in which the particles are spreading, but it does not lose the target object. Figure 8 shows the discriminable features enhanced from the 269th frame to the 302th frame, where the particles converge again around the 
centre of the target object after a few frames. Then, the tracker enters the leaves from the top and emerges from the bottom of the tree. The particle filter loses the target object under poor background conditions. Figure 9 shows the procedure on how the particles lose the target because of the simple sampling mechanism. Particles with high weight are chosen for state estimation, but they may be useless in a cluttered background that is not in the object region, and vice versa. When the tracker leaves the background region, it encounters the same static background. Thus, the object merges into the background. Our tracker treats the object and the background as a whole. When the tracker emerges from the same static background, the region widens and splits into two. Figure 10 shows the splitting procedure. Our tracker can recognize and determine the object well with velocity likelihood.

The comparable curve is plotted after tracing all the frames. Figures 11 and 12 show the $\mathrm{x}$-coordinate and y-coordinate curves, respectively. Figure 13 shows the Euclidean distance error curve of the three methods. Figures 11 and 12 show four lines corresponding to the ground truth, particle filter-geometric active contour (pf-gac), particle filter, and CamShift. The thick solid lines, which are referred to as 'lost' and correspond to pf and CamShift, indicate the frames that cannot be tracked. The abnormal error position is represented by the symbols e1, e2,..., e6. The symbols in Figure 13 are used to compare the abnormal and normal errors. In Figure 13, position e6 caused by the splitting of the particle from the background corresponds to the significant change in the x-coordinate in Figure 11 and the procedure in Figure 10. The positions e1 to e5 are caused by the inner color block splitting, as shown in Figure 14. This splitting is generated by the changes in pose and illumination as well as other reasons. Table 1 shows the tracking performance of the aforementioned methods. The pf-gac incurs the smallest average error and highest success ratio among the three methods. Thus, the pf-gac is more robust than the other two methods in a cluttered environment.

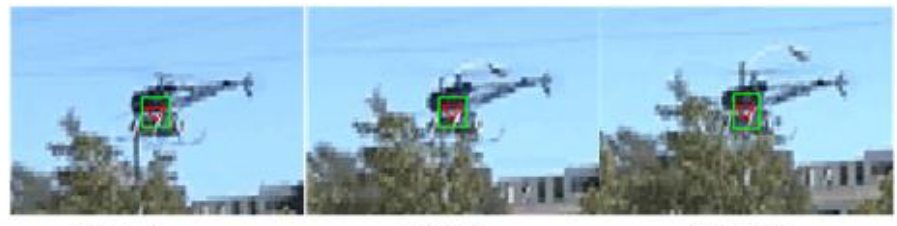
225th frame
230th frame
235th frame

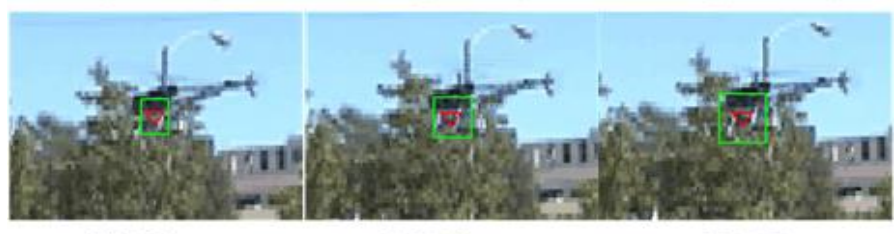

243th frame

247th frame

249th frame

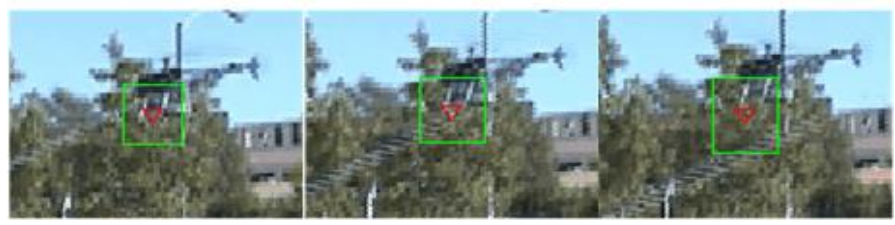

251th frame

252th frame

253th frame

Figure 7. CamShift Tracking Procedure 

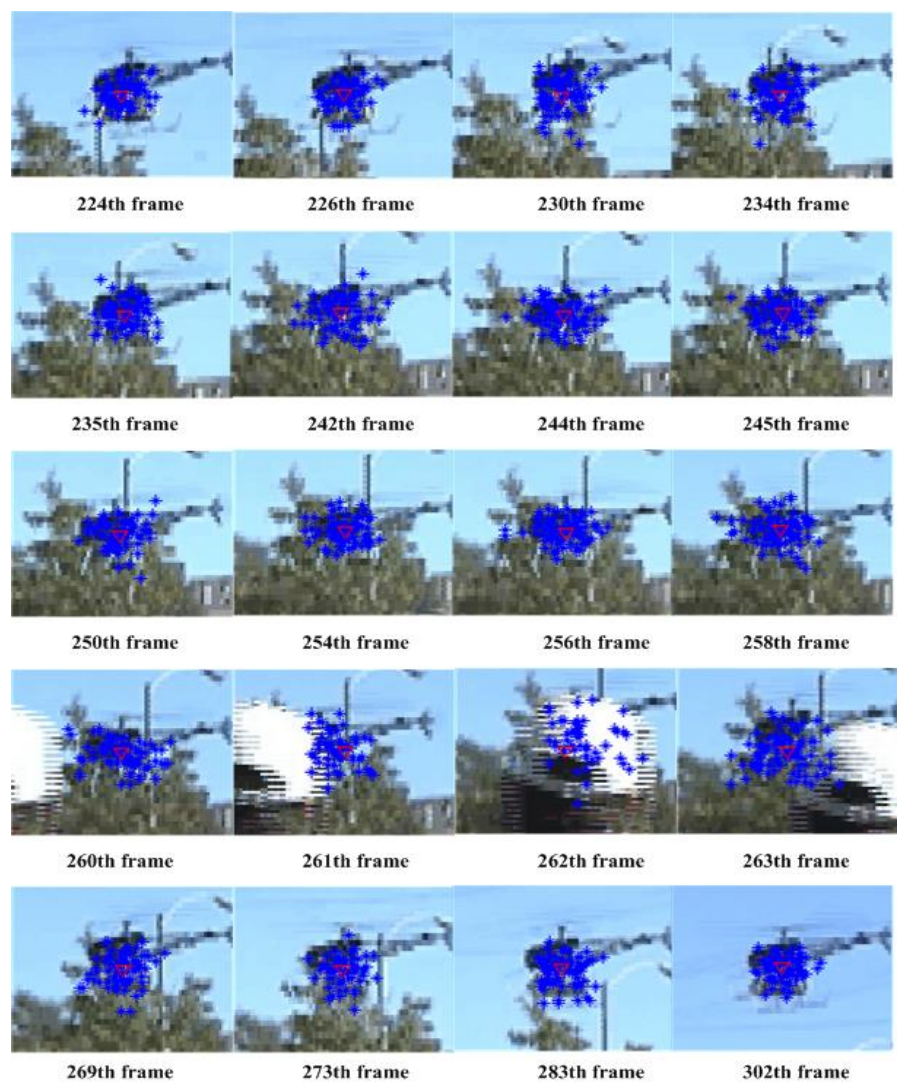

Figure 8. Tracking Procedure of Particle Filter until Converge Again

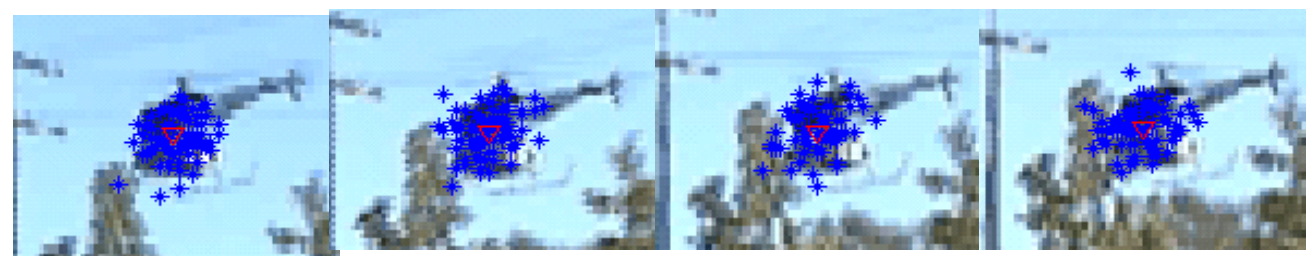

435 th frame

445 th frame

450th frame

455 th frame

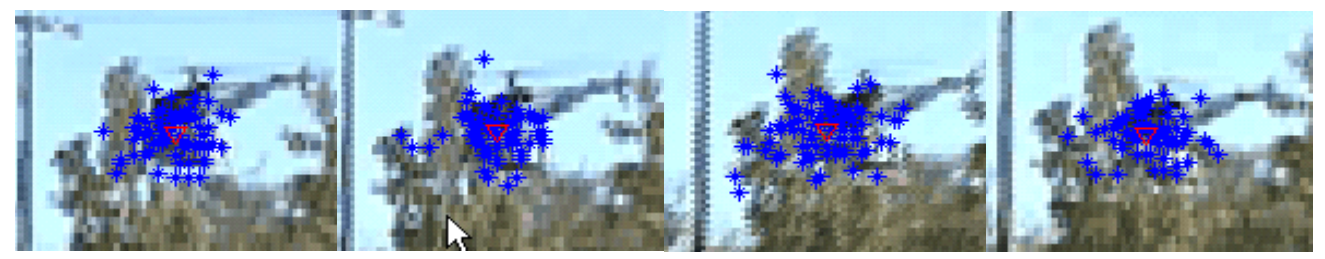

460 th frame

465 th frame 470 th frame

475 th frame

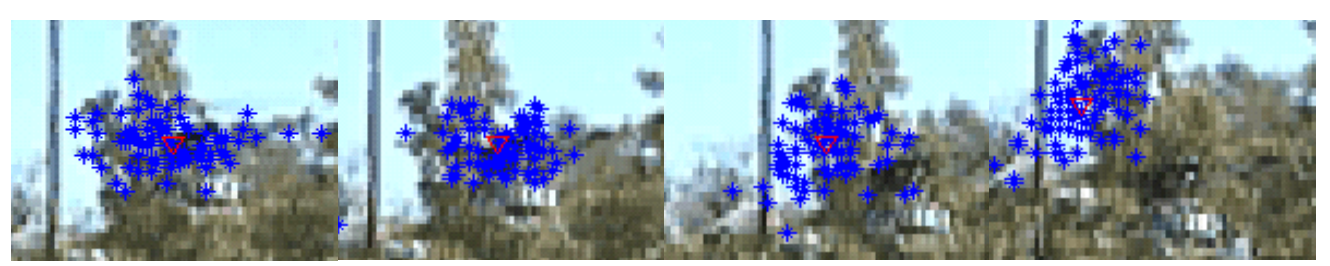

Figure 9. Tracking Procedure of Particle Filter until Lost 

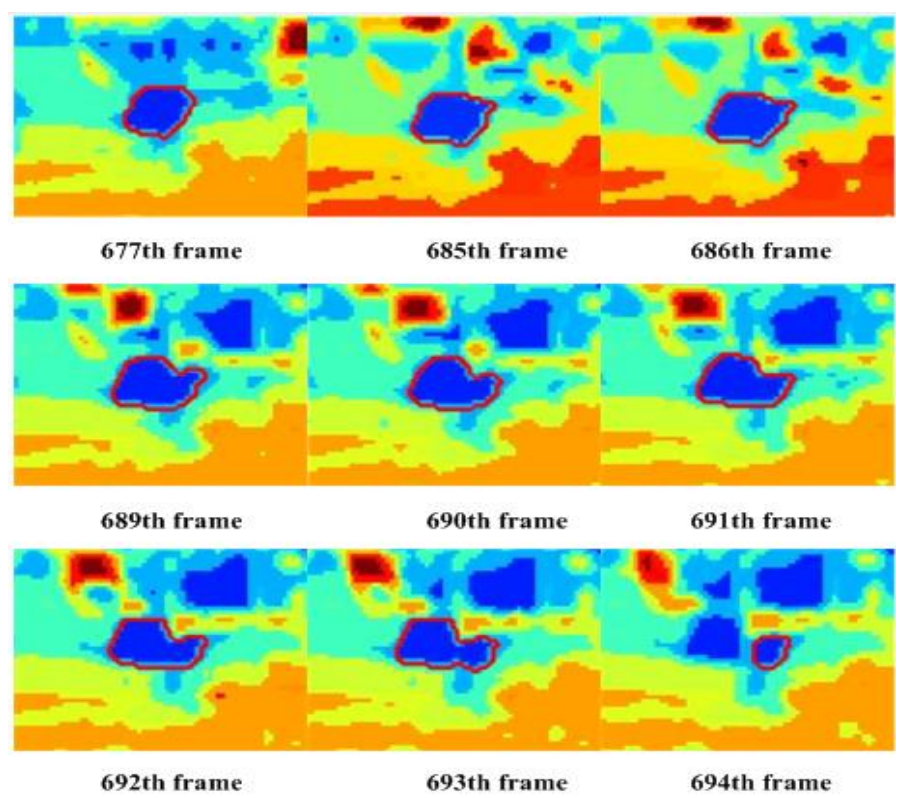

Figure 10. Object Splitting from Background

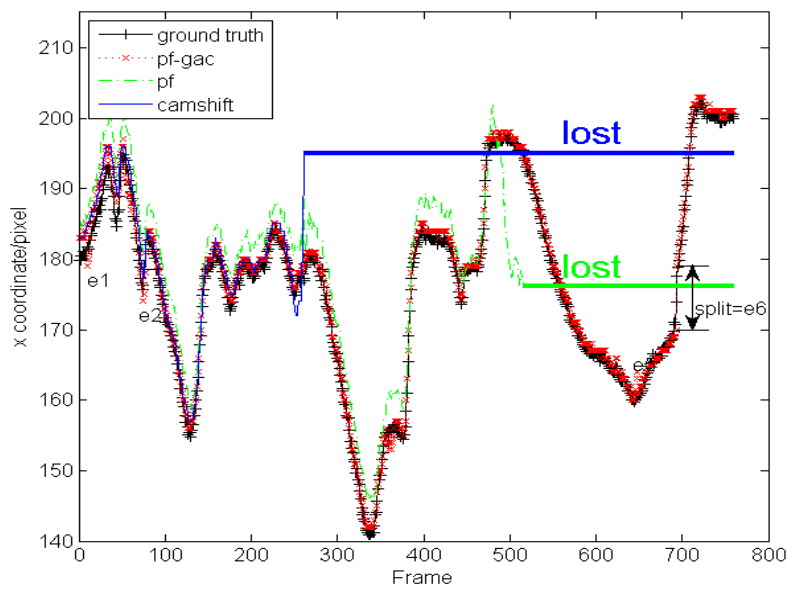

Figure 11. x Coordinate Position

Table 1. Comparable Performance of Different Algorithms

\begin{tabular}{llll}
\hline method & $\bar{Q}$ & $N_{\text {loss }}$ & $R_{\text {sucess }}$ \\
\hline Pf-gac & 1.3653 & 6 & $99.21 \%$ \\
pf & 4.9763 & 265 & $65.13 \%$ \\
camshift & 5.2028 & 507 & $33.23 \%$ \\
\hline
\end{tabular}




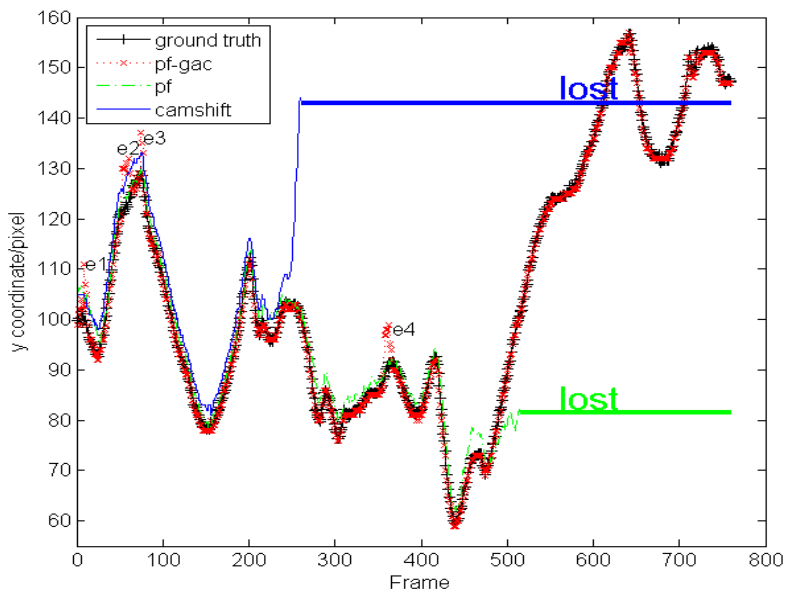

Figure 12. y Coordinate Position

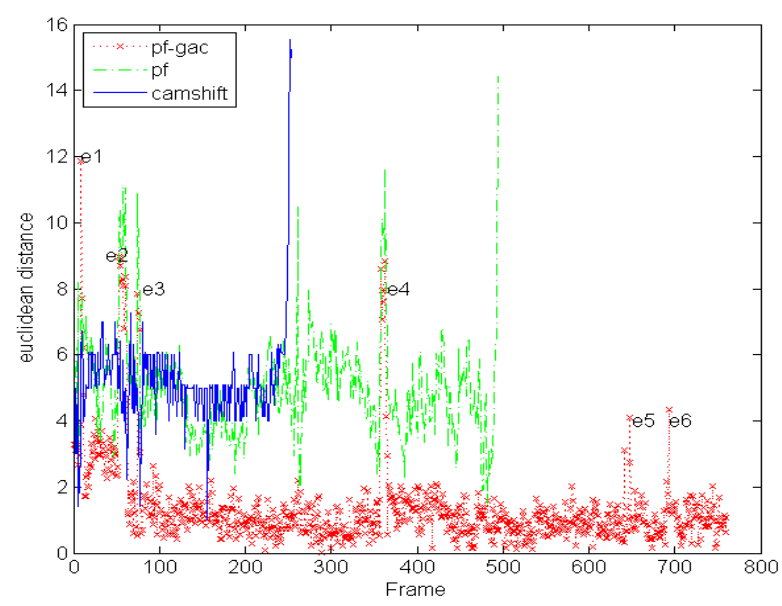

Figure 13. Euclidean Distance Error

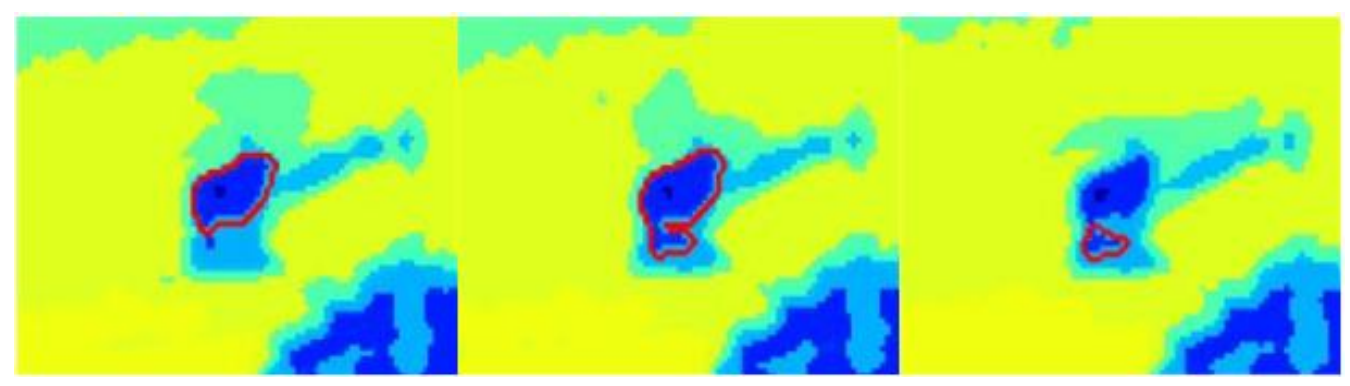

Figure 14. Object Inner Split

\section{Conclusion}

In this study, we proposed a novel robust object tracking algorithm called 'MutFilter'. Based on mutualism, this algorithm combines the use of the particle filter and the geometric active contour. MutFilter solves the curve initialization guess contour for active contour by weighted particles using the alpha shape method. It facilitates the approach of the initial 
contour to the real shape, thus making the curve evolution highly accurate and less time consuming, especially under scale changes and partial occlusion. We used the evolved curve contour to classify the particles, thus allowing the better representation of the target position by the useful particles than by the weight particles. We also employed the non-parameter-based resampling method to sample the particles and to improve diversity. The proposed algorithm combines the robustness of the particle filter with the flexibility of the active contour to improve the object state estimation. In this paper, we presented the detailed analyses and discussed the experiment procedure. The experimental results show that the proposed algorithm is highly robust in a cluttered environment.

\section{Acknowledgments}

The research work described in this paper is supported by national nature science foundation [No.50905136] and [No.51375368].The authors are grateful for the anonymous reviewers who made constructive comments.

\section{References}

[1] A. Yilmaz, O. Javed and M. Shah, "Object tracking: A survey", Acm Computing Surveys (CSUR), vol. 38, no. 4, (2006), pp. 1-45.

[2] M. Madhurima, "Object tracking in a video sequence using Mean-Shift Based Approach: An Implementation using MATLAB7", International Journal of Computational Engineering\&Management, vol. 11, no. 1, (2011), pp. 45-50.

[3] L. Mihaylova, P. Brasnett, N. Canagarajah and D. Bull, "Object tracking by particle filtering techniques in video sequences", Advances and Challenges in Multisensor Data and Information Processing, vol. 8, (2007), pp. 260-268.

[4] N. J. Gordon, D. J. Salmond and A. F. Smith, "Novel approach to nonlinear/non-Gaussian Bayesian state estimation", IEE Proceedings F (Radar and Signal Processing), vol. 140, no. 2, (1993), pp. 107-113.

[5] G. R. Bradski, "Computer vision face tracking for use in a perceptual user interface", Intel Technology Journal, vol. 2, no. 2, (1998), pp. 1-15.

[6] M. Kass, A. Witkin and D. Terzopoulos, "Snakes: Active contour models", International journal of computer vision, vol. 1, no. 4, (1988), pp. 321-331.

[7] T. F. Chan and L. A. Vese, "Active contours without edges", IEEE Transactions on Image Processing, vol. 10, no. 2, (2001), pp. 266-277.

[8] H. Shen, G. Nelson, S. Kennedy, D. Nelson, J. Johnson, D. Spiller, M. R. White and D. B. Kell, "Automatic tracking of biological cells and compartments using particle filters and active contours", Chemometrics and Intelligent Laboratory Systems, vol. 82, no. 1, (2006), pp. 276-282.

[9] Y. Rathi, N. Vaswani, A. Tannenbaum and A. Yezzi, "Tracking deforming objects using particle filtering for geometric active contours", IEEE Transactions on Pattern Analysis and Machine Intelligence, vol. 29, no. 8, (2007), pp. 1470-1475.

[10] X. Sun, H. Yao and S. Zhang, "A refined particle filter method for contour tracking", in SPIE International Conference on Visual Communications and Image Processing, (2010) July 11, Huang Shan, China.

[11] R. Sandhua, T. Georgioub and A. Tannenbauma, "Tracking with a New Distribution Metric in a Particle Filtering Framework", in Proc. of SPIE-IS\&T Electronic Imaging, CA, USA, (2008) February 26.

[12] J. MacCormick and M. Isard, "Partitioned sampling, articulated objects, and interface-quality hand tracking", in Proceedings of the 6th European Conference on Computer Vision, Dublin, Ireland, (2000) June 26-July 1.

[13] C. Luo, X. Cai and J. Zhang, "GATE:A Novel Robust Object Tracking Method Using the Particle Filtering and Level Set Method", in Digital Image Computing:Techniques and Applications, Canberra, Australia, (2008) December 1-3.

[14] H. Edelsbrunner, D. Kirkpatrick and R. Seidel, "On the shape of a set of points in the plane", IEEE Transactions on Information Theory, vol. 29, no. 4, (1983), pp. 551-559.

[15] N. Akkiraju, H. Edelsbrunner, M. Facello, P. Fu, E. Mücke and C. Varela, "Alpha shapes: definition and software", presented at the Proceedings of the 1st International Computational Geometry Software Workshop, Minneapolis, USA, (1995).

[16] P. J. Moran and M. Wagner, "Introducing alpha shapes for the analysis of path integral Monte Carlo results", in Proceedings of IEEE Conference on Visualization, Washington, DC, (1994) October 17-21.

[17] T. Chan and W. Zhu, "Level set based shape prior segmentation", in IEEE Computer Society Conference on Computer Vision and Pattern Recognition, SAN DIEGO,USA, (2005) June 20-25. 
[18] D. Cremers and S. Soatto, "A pseudo-distance for shape priors in level set segmentation", in 2nd IEEE workshop on variational, geometric and level set methods in computer vision, (2003) October, Nice, France.

[19] D. P. Huttenlocher, G. A. Klanderman, and W. J. Rucklidge, "Comparing images using the Hausdorff distance", IEEE Transactions on Pattern Analysis and Machine Intelligence, vol. 15, no. 9, (1993), pp. 850-863.

[20] X. Cai and A. Sowmya, "Level Learning Set: A Novel Classifier Based on Active Contour Models", in Proceedings of the18th European conference on Machine Learning, Warsaw, Poland, (2007) September 17-21.

[21] N. Kwak and C.-H. Choi, "Input feature selection by mutual information based on Parzen window", IEEE Transactions on Pattern Analysis and Machine Intelligence, vol. 24, no. 12, (2002), pp. 1667-1671.
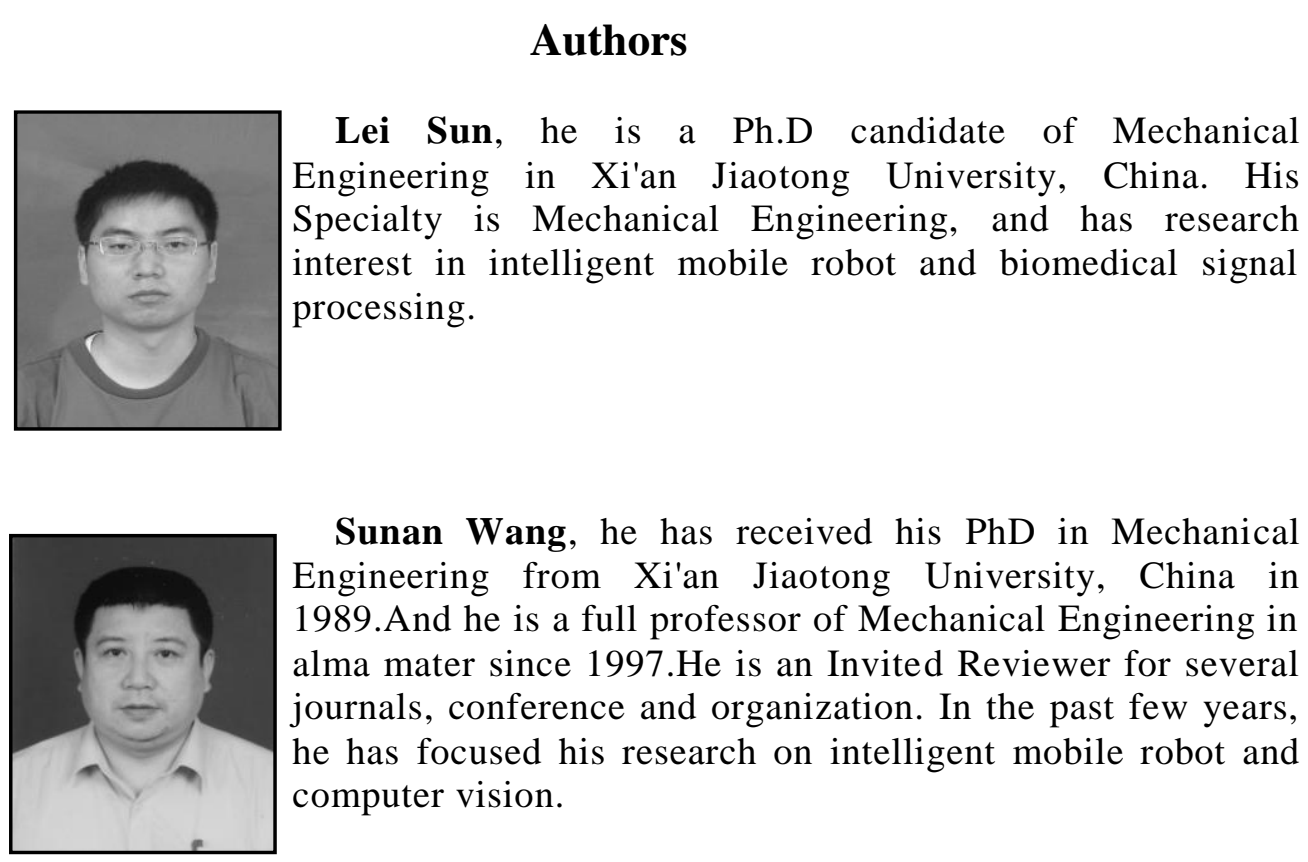

Sunan Wang, he has received his $\mathrm{PhD}$ in Mechanical Engineering from Xi'an Jiaotong University, China in 1989.And he is a full professor of Mechanical Engineering in alma mater since 1997.He is an Invited Reviewer for several journals, conference and organization. In the past few years, he has focused his research on intelligent mobile robot and computer vision.

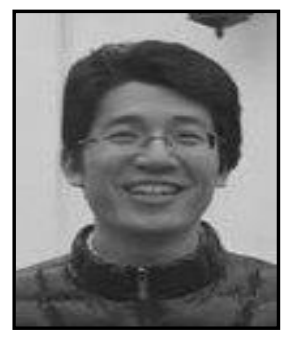

Jinhua Zhang, he is an Associate Professor in Xi'an Jiaotong University. He has received his $\mathrm{PhD}$ in Mechanical Engineering in 2008. His main research interests is intelligent robot based on biological signal.

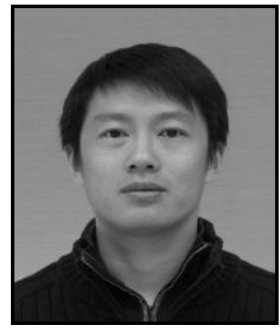

Xiaohu Li, he is a lecturer in school of mechanical engineering of Xi'an Jiaotong University. His primary research interests are in artificial intelligent, automatic control and machine tools. 